\title{
Nonconforming Finite Elements for the Stokes Problem*
}

\author{
By Michel Crouzeix and Richard S. Falk \\ Dedicated to Professor Eugene Isaacson on the occasion of his 70 th birthday
}

\begin{abstract}
A new stability result is obtained for the approximation of the stationary Stokes problem by nonconforming piecewise cubic approximations to the velocities and a discontinuous piecewise quadratic approximation to the pressure. The basic result is that for most reasonable meshes, these elements form a stable pair without the addition of quartic bubble functions (which had previously been added to insure stability).
\end{abstract}

1. Introduction. In the finite element approximation of the velocity-pressure formulation of the stationary Stokes equations using triangular finite elements, several approaches appear in the literature. One might classify these into conforming schemes which use a continuous piecewise polynomial approximation to the velocity and a discontinuous piecewise polynomial approximation to the pressure, conforming schemes which use continuous piecewise polynomial approximations for both velocity and pressure, macroelement schemes in which the pressure elements (usually discontinuous) are defined on a coarser mesh than the velocity elements, and nonconforming schemes in which the velocities are only continuous at appropriate Gauss points on the triangle edges, and discontinuous pressures are used.

In an early paper on the finite element approximation of the Stokes problem by the first author and P.-A. Raviart [4], several combinations of conforming and nonconforming velocity elements and discontinuous pressure elements were analyzed. Specifically, in the case of conforming velocity elements, it was shown that piecewise constant pressures could be paired with piecewise quadratic velocities (an idea suggested by Fortin [5]) to give a (suboptimal) $O(h)$ energy norm convergence rate. When discontinuous piecewise linear pressures are used, a corresponding velocity space of continuous piecewise quadratics augmented by cubic bubble functions improves the convergence rate to $O\left(h^{2}\right)$. In the case of discontinuous quadratic pressures, a corresponding velocity space of continuous piecewise cubics augmented by two quartic bubbles for each component of velocity gives an energy norm convergence rate of $O\left(h^{3}\right)$. In later work of Bernardi and Raugel [1], following another idea of Fortin [6], it was shown that pairing piecewise constant pressures with a velocity space consisting of continuous piecewise linear functions augmented by one (vector) quadratic per edge also yield an $O(h)$ energy norm convergence rate. In the case of higher-order elements, Scott and Vogelius [10] proved that except for some exceptional meshes, the use of discontinuous piecewise polynomials of degree

Received June 13, 1988.

1980 Mathematics Subject Classification (1985 Revision). Primary 65N30.

Key words and phrases. Stokes, finite element, nonconforming.

*This research was supported by NSF grant DMS-87-03354 (RSF). 
$n(n \geq 3)$ for pressure and continuous piecewise polynomials of degree $n+1$ for velocity (without adding any bubble functions) give methods with optimal-order convergence rates.

Since the pairing of constant pressures with continuous piecewise linear velocities is not convergent (for most meshes, the set of such velocities with zero divergence is $\{0\}$ ) and the substitution of quadratic velocities leads to a suboptimal convergence rate, Crouzeix and Raviart also studied the use of nonconforming velocity spaces. They showed that constant pressures paired with nonconforming piecewise linear velocities give an optimal $O(h)$ energy norm convergence rate. The case of nonconforming quadratic velocities was not considered in their paper, but was considered in a later paper of Fortin [7], where it was proved that nonconforming quadratic velocities may be paired with discontinuous linear pressures to produce an $O\left(h^{2}\right)$ method. Crouzeix and Raviart also considered the case of nonconforming cubic velocities and discontinuous quadratic pressures, but obtained an $O\left(h^{3}\right)$ convergence rate only by augmenting each component of the velocity space by two quartic bubble functions (as in the conforming case). The purpose of this paper is to improve this last result by showing that for most commonly used meshes, the quartic bubble functions are not needed to give a convergent method of optimal order. In light of the work of Scott and Vogelius (mentioned above) that conforming velocity elements of degree $\geq 4$ already give optimal-order methods, the three lowest-order cases of nonconforming elements appear to be the only ones of interest.

An outline of this paper is as follows. In Section 2 we define the notation to be used and recall some of the theory of the finite element approximation of the stationary Stokes equations. In the now standard approach to this problem, the essential feature of the analysis is the verification of an appropriate form of the infsup condition. The verification of such a condition for the choice of nonconforming cubic velocities and discontinuous quadratic pressures is done in the remaining sections for various types of meshes.

2. Notation and Preliminaries. For $\Omega$ a polygonal domain in $\mathbb{R}^{2}$, we thus consider the approximation of the stationary Stokes problem: Find $\mathbf{u}=\left(u_{1}, u_{2}\right)$ and $p$ satisfying

$$
\begin{aligned}
-\nu \Delta \mathbf{u}+\nabla p=\mathbf{f} & \text { in } \Omega, \\
\operatorname{div} \mathbf{u}=0 & \text { in } \Omega, \\
\mathbf{u}=0 & \text { on } \partial \Omega,
\end{aligned}
$$

where $\mathbf{u}$ is the fluid velocity, $p$ is the pressure, $\mathbf{f}$ are the body forces per unit mass, and $\nu$ is the viscosity. The variational formulation of this problem is:

Find $\mathbf{u}=\left(u_{1}, u_{2}\right) \in\left(H_{0}^{1}(\Omega)\right)^{2}$ and $p \in L^{2}(\Omega) / \mathbb{R}$ satisfying

$$
\begin{aligned}
a(\mathbf{u}, \mathbf{v})-(p, \operatorname{div} \mathbf{v}) & =(\mathbf{f}, \mathbf{v}) & & \text { for all } \mathbf{v} \in\left(H_{0}^{1}(\Omega)\right)^{2} \\
(\operatorname{div} \mathbf{u}, q) & =0 & & \text { for all } q \in L^{2}(\Omega) / \mathbb{R}
\end{aligned}
$$

where

$$
a(\mathbf{u}, \mathbf{v})=\nu \sum_{i, j=1}^{2} \int_{\Omega} \frac{\partial u_{i}}{\partial x_{j}} \frac{\partial v_{i}}{\partial x_{j}} d x
$$

and $(\cdot, \cdot)$ denotes the $L^{2}(\Omega)$ inner product. 
The finite element methods we consider may be described abstractly as follows. We let $\tau_{h}$ denote a triangulation of $\Omega$ by triangles $T$ of diameter $\leq h$. We then denote by $\mathbf{W}_{h}$ a finite-dimensional approximation of $\left(H_{0}^{1}(\Omega)\right)^{2}$. Since we are considering nonconforming methods, $\mathbf{W}_{h} \nsubseteq\left(H_{0}^{1}(\Omega)\right)^{2}$, but $\left.\mathbf{v}_{h}\right|_{T} \in H^{1}(T)$ for all $\mathbf{v}_{h} \in \mathbf{W}_{h}$ and $T \in \tau_{h}$. We assume that

$$
\left\|\mathbf{v}_{h}\right\|_{1, h}=\left(\sum_{T \in \tau_{h}}\left|\mathbf{v}_{h}\right|_{1, T}^{2}\right)^{1 / 2}
$$

is a norm on $\mathbf{W}_{h}$. Let $Q_{h}$ denote a finite-dimensional subspace of $L^{2}(\Omega) / \mathbb{R}$. The approximation scheme is then: Find $\mathbf{u}_{h} \in \mathbf{W}_{h}, p_{h} \in Q_{h}$ satisfying

$$
\begin{aligned}
a_{h}\left(\mathbf{u}_{h}, \mathbf{v}_{h}\right)-\left(p_{h}, \operatorname{div}_{h} \mathbf{v}_{h}\right) & =\left(\mathbf{f}, \mathbf{v}_{h}\right) & & \text { for all } \mathbf{v}_{h} \in \mathbf{W}_{h}, \\
\left(\operatorname{div}_{h} \mathbf{u}_{h}, q\right) & =0 & & \text { for all } q \in Q_{h},
\end{aligned}
$$

where

$$
a_{h}(\mathbf{u}, \mathbf{v})=\nu \sum_{T \in \tau_{h}} \sum_{i, j=1}^{2} \int_{T} \frac{\partial u_{i}}{\partial x_{j}} \frac{\partial v_{i}}{\partial x_{j}} d x
$$

and $\operatorname{div}_{h} \mathbf{v}$ is the $L^{2}(\Omega)$ function whose restriction to each triangle $T \in \tau_{h}$ is given by $\left.(\operatorname{div} \mathbf{v})\right|_{T}$.

The analysis of this type of method is well understood. If the space $\mathbf{W}_{h}$ were conforming, the general theory of saddle-point problems developed by Babuskka and Brezzi could be applied directly. In that case the only difficulty in the analysis is the verification of the inf-sup condition

$$
\inf _{0 \neq q_{h} \in Q_{h}} \sup _{0 \neq \mathbf{v}_{h} \in \mathbf{W}_{h}} \frac{\left(\operatorname{div} \mathbf{v}_{h}, q_{h}\right)}{\left\|\mathbf{v}_{h}\right\|_{1}\left\|q_{h}\right\|_{0}} \geq \gamma .
$$

When (2.1) holds, one obtains the quasi-optimal error estimate

$$
\left\|\mathbf{u}-\mathbf{u}_{h}\right\|_{1}+\left\|p-p_{h}\right\|_{0} \leq C \inf \left(\left\|\mathbf{u}-\mathbf{v}_{h}\right\|_{1}+\left\|p-q_{h}\right\|_{0}\right),
$$

where the inf is taken over all $\mathbf{v}_{h} \in \mathbf{W}_{h}$ and $q_{h} \in Q_{h}$. For nonconforming methods, a straightforward modification of this result leads to the conclusion that if

$$
\inf _{0 \neq q_{h} \in Q_{h}} \sup _{0 \neq \mathbf{v}_{h} \in \mathbf{W}_{h}} \frac{\left(\operatorname{div}_{h} \mathbf{v}_{h}, q_{h}\right)}{\left\|\mathbf{v}_{h}\right\|_{1, h}\left\|q_{h}\right\|_{0}} \geq \gamma
$$

then one obtains the error estimate

$$
\begin{aligned}
\| \mathbf{u}- & \mathbf{u}_{h}\left\|_{1, h}+\right\| p-p_{h} \|_{0} \\
& \leq C\left[\inf \left(\left\|\mathbf{u}-\mathbf{v}_{h}\right\|_{1, h}+\left\|p-q_{h}\right\|_{0}\right)+\sup \frac{\sum_{T} \int_{\partial T}\left(\nu \frac{\partial \mathbf{u}}{\partial n}-p \mathbf{n}\right) \cdot \mathbf{w}_{h}}{\left\|\mathbf{w}_{h}\right\|_{1, h}}\right],
\end{aligned}
$$

where the inf is taken over all $\mathbf{v}_{h} \in \mathbf{W}_{h}$ and $q_{h} \in Q_{h}$ and the sup is taken over all $\mathbf{w}_{h} \in \mathbf{W}_{h}$.

Since [4] predated the work of Brezzi [3], the analysis in the former paper does not proceed by giving a direct verification of condition (2.2). However, using the interpolants constructed in [4], condition (2.2) can be easily verified on each triangle for the elements considered, with the global result following immediately. This local verification of condition (2.2) depends on the fact that appropriate bubble 
functions have been added to the basic nonconforming spaces. In order to verify (2.2) without adding these bubble functions, we instead seek to verify (2.2) first on a patch of elements. This idea was previously used by Boland and Nicolaides [2] and Stenberg [11]. The basic technique is described in Girault and Raviart [8, pp. 129-132] for the case of conforming elements. In our case, we first define a collection of subdomains $\Omega_{r} \subset \Omega, r=1,2, \ldots, R$, such that $\bar{\Omega}_{r}$ is a union of triangles $T_{0, r}, T_{1, r}, \ldots, T_{M_{r}, r} \in \tau_{h}$ and

$$
\bar{\Omega}=\bigcup_{r=1}^{R} \bar{\Omega}_{r}
$$

We assume that there exists a constant $L$ independent of $h$ such that for all $T \in \tau_{h}$ the number of $r$ such that $\Omega_{r} \cap T \neq \varnothing$ is bounded by $L$. We next define

$$
\begin{gathered}
\mathbf{V}_{h}\left(\Omega_{r}\right)=\left\{\mathbf{u}_{h}:\left.\mathbf{u}_{h}\right|_{T_{k, r}} \in\left(P_{3}\right)^{2}, k=0,1, \ldots, M_{r}, \mathbf{u}_{h}=0 \text { in } \Omega / \Omega_{r},\right. \\
\left.\mathbf{u}_{h} \text { continuous at the Gauss points on all triangle sides }\right\}, \\
H_{h}\left(\Omega_{r}\right)=\left\{q \in L^{2}\left(\Omega_{r}\right):\left.q\right|_{T_{k, r}} \in P_{2} \text { and } \int_{T_{k, r}} q d x=0,\right. \\
\left.k=0,1, \ldots, M_{r}, q=0 \text { in } \Omega / \Omega_{r}\right\} .
\end{gathered}
$$

The weak local verification of condition (2.2) on the patch of elements $\Omega_{r}$ will consist of establishing the validity of the following hypothesis:

Hypothesis H0. There exists a positive constant $\gamma^{*}$, independent of $h$ and $r$, such that

$$
\inf _{0 \neq q_{h} \in H_{h}} \sup _{0 \neq \mathbf{v}_{h} \in \mathbf{V}_{h}} \frac{\left(\operatorname{div}_{h} \mathbf{v}_{h}, q_{h}\right)}{\left\|\mathbf{v}_{h}\right\|_{1, h, \Omega_{r}}\left\|q_{h}\right\|_{0, \Omega_{r}}} \geq \gamma^{*} .
$$

We shall do this by showing the validity of

Hypothesis $\mathrm{H} 1$. For all $q_{h} \in H_{h}\left(\Omega_{r}\right)$, there exists $\mathbf{u}_{h} \in \mathbf{V}_{h}\left(\Omega_{r}\right)$ such that

$$
\operatorname{div}_{h} \mathbf{u}_{h}=q_{h} \text { and }\left\|\mathbf{u}_{h}\right\|_{1, h, \Omega_{r}} \leq\left\|q_{h}\right\|_{0, \Omega_{r}} / \gamma^{*} .
$$

As in the papers mentioned above (e.g. see Theorem 1.12 of [8]), we can establish the following result. We include a proof for the sake of completeness and also since we do not assume, as in [2] and [8], that the domains $\Omega_{r}$ are disjoint.

THEOREM 2.1. Let

$$
\begin{aligned}
& \mathbf{W}_{h}=\left\{\mathbf{u}_{h}:\left.\mathbf{u}_{h}\right|_{T} \in\left(P_{3}\right)^{2}, T \in \tau_{h}, \mathbf{u}_{h}\right. \text { continuous at the Gauss points } \\
&\text { on all triangle sides, } \left.\mathbf{u}_{h}=0 \text { at the Gauss points on } \partial \Omega\right\}, \\
& Q_{h}=\left\{q \in L^{2}(\Omega),\left.q\right|_{T} \in P_{2}, T \in \tau_{h}, \int_{\Omega} q d x=0\right\} .
\end{aligned}
$$

Then, if hypothesis $\mathrm{H} 1$ is satisfied, condition (2.2) holds with a constant $\gamma$ independent of $h$.

Proof. For $q_{h} \in Q_{h}$, one can construct by standard techniques a function $\mathbf{w}_{h} \in$ $\mathrm{W}_{h}$ satisfying for all $T \in \tau_{h}$ the conditions

$$
\int_{T} \operatorname{div}_{h} \mathbf{w}_{h} d x=\int_{T} q_{h} d x, \quad\left\|\mathbf{w}_{h}\right\|_{1, h, \Omega} \leq\left\|q_{h}\right\|_{0, \Omega} / \gamma_{1},
$$


with $\gamma_{1}$ independent of $h$. Setting

$$
p_{h}=q_{h}-\operatorname{div}_{h} \mathbf{w}_{h},
$$

we may write

$$
p_{h}=\sum_{r=1}^{R} p_{h, r}
$$

where

$$
p_{h, r} \in H_{h}\left(\Omega_{r}\right) \quad \text { and } \quad p_{h, r} \cdot p_{h, r^{\prime}}=0 \text { for } r \neq r^{\prime} .
$$

Hence we have

$$
\left\|p_{h}\right\|_{0, \Omega}^{2}=\sum_{r=1}^{R}\left\|p_{h, r}\right\|_{0, \Omega_{r}}^{2} .
$$

Using (2.5), we may write

$$
p_{h, r}=\operatorname{div}_{h} \mathbf{u}_{h, r} \quad \text { with }\left\|\mathbf{u}_{h, r}\right\|_{1, h, \Omega_{r}} \leq\left\|p_{h, r}\right\|_{0, \Omega_{r}} / \gamma^{*} .
$$

Then we set

$$
\mathbf{u}_{h}=\sum_{r=1}^{R} \mathbf{u}_{h, r} \quad \text { and } \quad \mathbf{v}_{h}=\mathbf{u}_{h}+\mathbf{w}_{h}
$$

so that we have

$$
\operatorname{div}_{h} \mathbf{v}_{h}=q_{h}
$$

and

$$
\begin{aligned}
\left\|\mathbf{u}_{h}\right\|_{1, h, \Omega}^{2} & =\sum_{T \in \tau_{h}}\left\|\mathbf{u}_{h}\right\|_{1, T}^{2} \leq L \sum_{T \in \tau_{h}} \sum_{r=1}^{R}\left\|\mathbf{u}_{h, r}\right\|_{1, T}^{2} \\
& \leq L \sum_{r=1}^{R}\left\|\mathbf{u}_{h, r}\right\|_{1, h, \Omega_{r}}^{2} \leq L\left\|p_{h}\right\|_{0, \Omega}^{2} / \gamma^{* 2}
\end{aligned}
$$

From (2.6) it follows that

$$
\begin{aligned}
\left\|p_{h}\right\|_{0, \Omega} & \leq\left\|q_{h}\right\|_{0, \Omega}+\left\|\operatorname{div}_{h} \mathbf{w}_{h}\right\|_{0, \Omega} \leq\left\|q_{h}\right\|_{0, \Omega}+\sqrt{2}\left\|\mathbf{w}_{h}\right\|_{1, h, \Omega} \\
& \leq\left(1+\sqrt{2} / \gamma_{1}\right)\left\|q_{h}\right\|_{0, \Omega} .
\end{aligned}
$$

Using (2.6) and (2.7), we then obtain

$$
\left\|\mathbf{v}_{h}\right\|_{1, h, \Omega} \leq\left\|\mathbf{u}_{h}\right\|_{1, h, \Omega}+\left\|\mathbf{w}_{h}\right\|_{1, h, \Omega} \leq \sqrt{L}\left(\gamma_{1}+\sqrt{2}\right)\left\|q_{h}\right\|_{0, \Omega} /\left(\gamma^{*} \gamma_{1}\right)+\left\|q_{h}\right\|_{0, \Omega} / \gamma_{1} .
$$

Finally,

$$
\left\|\mathbf{v}_{h}\right\|_{1, h, \Omega} \leq\left\|q_{h}\right\|_{0, \Omega} / \gamma
$$

where

$$
\gamma=\gamma^{*} \gamma_{1} /\left[\sqrt{L}\left(\gamma_{1}+\sqrt{2}\right)+\gamma^{*}\right]
$$

Together with (2.8), this implies (2.2).

It then follows directly from (2.3) and standard estimates for nonconforming methods (see [4] and [9]) that if $\mathbf{u} \in\left(H^{4}(\Omega)\right)^{2}$ and $p \in H^{3}(\Omega)$, then

$$
\left\|\mathbf{u}-\mathbf{u}_{h}\right\|_{1, h}+\left\|p-p_{h}\right\|_{0} \leq C h^{3}\left(\|\mathbf{u}\|_{4}+\|p\|_{3}\right) \text {. }
$$


Thus, to establish Theorem 2.1 and the error estimate (2.9), we need only show that Hypothesis $\mathrm{H} 1$ is valid. Before doing so in the next sections, we first state some basic facts that will facilitate the forthcoming analysis. We recall that the barycentric coordinates $\lambda_{i}=\lambda_{i}(\mathbf{x}), 1 \leq i \leq 3$, of any point $\mathbf{x} \in \mathbb{R}^{2}$ with respect to the points $A_{1}, A_{2}, A_{3}$ are the unique solutions of the linear system

$$
\sum_{j=1}^{3} A_{j} \lambda_{j}=\mathbf{x}
$$

$$
\sum_{j=1}^{3} \lambda_{j}=1
$$

Denoting by $\overrightarrow{A_{1} A_{2}}$ the vector from $A_{1}$ to $A_{2}$, we have

$$
\mathbf{x}=A_{1}+\lambda_{2}{\overrightarrow{A_{1} A}}_{2}+\lambda_{3}{\overrightarrow{A_{1} A_{3}}}_{\text {. }}
$$

Hence,

$$
I=\nabla \mathbf{x}^{t}=\nabla \lambda_{2}\left({\overrightarrow{A_{1} A_{2}}}^{t}+\nabla \lambda_{3}\left({\overrightarrow{A_{1} A_{3}}}^{t}\right.\right.
$$

and for any vector $\mathbf{u}$,

$$
\mathbf{u}=\left(\mathbf{u} \cdot \nabla \lambda_{2}\right){\overrightarrow{A_{1} A_{2}}}+\left(\mathbf{u} \cdot \nabla \lambda_{3}\right){\overrightarrow{A_{1} A_{3}}}
$$

By applying (2.13) to the vector $\nabla \lambda_{2}$, it easily follows that

$$
\nabla \lambda_{2} \cdot{\overrightarrow{A_{1} A_{2}}}_{2}=1 \text { and } \nabla \lambda_{2} \cdot \overrightarrow{A_{1} A_{3}}=0
$$

3. Verification of Hypothesis $\mathbf{H} 1$-Mesh $\mathbf{I}$. The verification of condition (2.5) will be done for three choices of the subdomain $\Omega_{r}$. In the first of these, $\Omega_{r}=T_{0} \cup T_{1} \cup T_{2} \cup T_{3}$, where the four triangles $T_{0}, T_{1}, T_{2}, T_{3}$ are aligned in the configuration depicted in Figure 3.1.

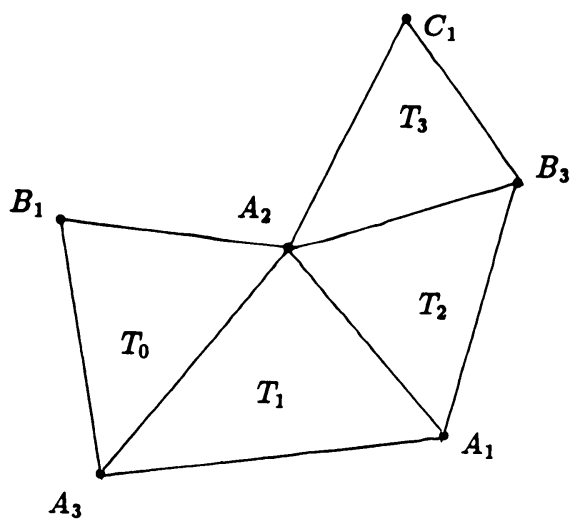

FIGURE 3.1 
We denote by

$\left(\mu_{1}, \mu_{2}, \mu_{3}\right)$ the barycentric coordinates of $B_{3}$ with respect to $A_{1}, A_{2}, A_{3}$,

$\left(\nu_{1}, \nu_{2}, \nu_{3}\right)$ the barycentric coordinates of $B_{1}$ with respect to $A_{1}, A_{2}, A_{3}$,

$\left(\tau_{1}, \tau_{2}, \tau_{3}\right)$ the barycentric coordinates of $C_{1}$ with respect to $A_{1}, A_{2}, B_{3}$.

The verification of Hypothesis $\mathrm{H} 1$ will depend on a geometrical condition on the mesh patch $\Omega_{r}$ given in terms of the barycentric coordinates defined above. Following the verification, we describe some common situations which are and are not covered. To simplify the proof of our result, it is convenient to first define the following subspace of $\mathbf{V}_{h}\left(\Omega_{r}\right)$. Let

$$
\hat{\mathbf{V}}_{h}\left(\Omega_{r}\right)=\left\{\mathbf{u}_{h} \in \mathbf{V}_{h}\left(\Omega_{r}\right): \int_{s} \mathbf{u}_{h} d \sigma=0 \text { for all sides } s \text { of } \Omega_{r}\right\} .
$$

The verification of condition (2.5) is contained in the following proposition.

PROPOSITION 3.1. If $D \equiv-\nu_{2} \tau_{2} \mu_{1}^{2}-\left(\nu_{1}-1\right) \tau_{2} \mu_{2} \mu_{3}+\left(\tau_{1}-1\right) \nu_{2} \mu_{2} \neq 0$, then the operator $\operatorname{div}_{h}$ from $\hat{\mathbf{V}}_{h}$ into $H_{h}$ is one-to-one and for all $\mathbf{u}_{h} \in \hat{\mathbf{V}}_{h}$

$$
\left\|\mathbf{u}_{h}\right\|_{1, h, \Omega_{r}} \leq \frac{C(\theta)}{|D|}\left\|\operatorname{div}_{h} \mathbf{u}_{h}\right\|_{0, \Omega_{r}}
$$

where $C$ is a constant which depends only on the smallest angle $\theta$ of the triangles $T_{0}, T_{1}, T_{2}$, and $T_{3}$.

Proof. First we remark that $\mathbf{u}_{h} \in \hat{\mathbf{V}}_{h}$ implies $\int_{T_{k}} \operatorname{div}_{h} \mathbf{u}_{h} d x=0$, so that $\operatorname{div}_{h}\left(\hat{\mathbf{V}}_{h}\right) \subseteq H_{h}$. Since $\operatorname{dim} \hat{\mathbf{V}}_{h}=\operatorname{dim} H_{h}=20, \operatorname{div}_{h}$ is one-to-one if and only if $\operatorname{Ker}\left(\operatorname{div}_{h}\right)=\{0\}$.

In the triangle $T_{k}$, we may write

$$
\begin{aligned}
\mathbf{u}_{h} & =\sum_{i=1}^{3} \mathbf{u}_{i}^{(k)} \lambda_{i}^{3}+\sum_{i \neq j} \mathbf{u}_{i j}^{(k)} \lambda_{i}^{2} \lambda_{j}+\mathbf{u}_{123}^{(k)} \lambda_{1} \lambda_{2} \lambda_{3}, \\
q & =\sum_{i=1}^{3} q_{i}^{(k)} \lambda_{i}^{2}+\sum_{i<j} q_{i j}^{(k)} \lambda_{i} \lambda_{j},
\end{aligned}
$$

where $\lambda_{i}=\lambda_{i}^{(k)}$ are the barycentric coordinates in the triangle $T_{k}$. We shall omit the superscript $(k)$ when there is no ambiguity.

We now turn to the lengthy process of expressing the continuity of $\mathbf{u}_{h}$ at the Gauss points and the satisfaction of the equations $\operatorname{div}_{h} \mathbf{u}_{h}=q$ on each triangle $T$ and $\int_{s} \mathbf{u}_{h} d \sigma=0$ for each triangle side $s$, in terms of the degrees of freedom of $\mathbf{u}_{h}$ and $q$. On the side ${\overrightarrow{A_{2}}}_{3}$ or ${\overrightarrow{A_{2} B}}_{3}$, the condition $\int_{s} \mathbf{u}_{h} d \sigma=0$ becomes

$$
3\left(\mathbf{u}_{2}+\mathbf{u}_{3}\right)+\left(\mathbf{u}_{23}+\mathbf{u}_{32}\right)=0 .
$$

Similarly, we have on ${\overrightarrow{A_{2} B_{1}}}_{\overrightarrow{A_{2} A_{1}}},{\overrightarrow{A_{2} C_{1}}}$

$$
3\left(\mathbf{u}_{2}+\mathbf{u}_{1}\right)+\left(\mathbf{u}_{21}+\mathbf{u}_{12}\right)=0 .
$$

The continuity at the Gauss points on $\vec{A}_{2} A_{3}$ (barycentric coordinates $(0,1 / 2$, $1 / 2),(0, \theta, 1-\theta),(0,1-\theta, \theta)$ with $\theta(1-\theta)=1 / 10)$ gives

$$
\left(\mathbf{u}_{2}+\mathbf{u}_{3}+\mathbf{u}_{23}+\mathbf{u}_{32}\right)^{(0)}=\left(\mathbf{u}_{2}+\mathbf{u}_{3}+\mathbf{u}_{23}+\mathbf{u}_{32}\right)^{(1)},
$$




$$
\begin{aligned}
& \left(\theta^{3} \mathbf{u}_{2}+(1-\theta)^{3} \mathbf{u}_{3}+\theta(1-\theta)\left[\theta \mathbf{u}_{23}+(1-\theta) \mathbf{u}_{32}\right]\right)^{(0)} \\
& \quad=\left(\theta^{3} \mathbf{u}_{2}+(1-\theta)^{3} \mathbf{u}_{3}+\theta(1-\theta)\left[\theta \mathbf{u}_{23}+(1-\theta) \mathbf{u}_{32}\right]\right)^{(1)} \\
& \left([1-\theta]^{3} \mathbf{u}_{2}+\theta^{3} \mathbf{u}_{3}+\theta(1-\theta)\left[(1-\theta) \mathbf{u}_{23}+\theta \mathbf{u}_{32}\right]\right)^{(0)} \\
& \quad=\left([1-\theta]^{3} \mathbf{u}_{2}+\theta^{3} \mathbf{u}_{3}+\theta(1-\theta)\left[(1-\theta) \mathbf{u}_{23}+\theta \mathbf{u}_{32}\right]\right)^{(1)}
\end{aligned}
$$

Since $\theta(1-\theta)=1 / 10$ implies $\theta^{2}=\theta-1 / 10$ and $\theta^{3}=(9 \theta-1) / 10$, we have

$$
\begin{aligned}
\theta^{3} \mathbf{u}_{2}+ & (1-\theta)^{3} \mathbf{u}_{3}+\theta(1-\theta)\left[\theta \mathbf{u}_{23}+(1-\theta) \mathbf{u}_{32}\right] \\
= & \left\{(9 \theta-1) \mathbf{u}_{2}+(8-9 \theta) \mathbf{u}_{3}+\left[\theta \mathbf{u}_{23}+(1-\theta) \mathbf{u}_{32}\right]\right\} / 10 \\
= & \left\{(1-\theta)\left[3\left(\mathbf{u}_{2}+\mathbf{u}_{3}\right)+\mathbf{u}_{23}+\mathbf{u}_{32}\right]+(2 \theta-1)\left(9 \mathbf{u}_{2}+\mathbf{u}_{23}\right)\right. \\
& \left.+(5-6 \theta)\left(\mathbf{u}_{2}+\mathbf{u}_{3}\right)\right\} / 10 .
\end{aligned}
$$

Using an analogous rewriting of Eq. (3.5) and taking into account Eq. (3.1), we have the continuity at the Gauss points on ${\overrightarrow{A_{2} A_{3}}}_{3}$ and ${\overrightarrow{A_{2} B_{3}}}_{3}$ if and only if

$$
\begin{aligned}
& \left(\mathbf{u}_{2}+\mathbf{u}_{3}\right)^{(k)}=\left(\mathbf{u}_{2}+\mathbf{u}_{3}\right)^{(k+1)}, \quad k=0,2, \\
& \left(9 \mathbf{u}_{2}+\mathbf{u}_{23}\right)^{(k)}=\left(9 \mathbf{u}_{2}+\mathbf{u}_{23}\right)^{(k+1)}, \quad k=0,2 .
\end{aligned}
$$

Similarly, continuity at the Gauss points on $\overrightarrow{A_{2} A_{1}}$ becomes

$$
\begin{gathered}
\left(\mathbf{u}_{1}+\mathbf{u}_{2}\right)^{(1)}=\left(\mathbf{u}_{1}+\mathbf{u}_{2}\right)^{(2)}, \\
\left(9 \mathbf{u}_{1}+\mathbf{u}_{12}\right)^{(1)}=\left(9 \mathbf{u}_{1}+\mathbf{u}_{12}\right)^{(2)} .
\end{gathered}
$$

The continuity with 0 at the Gauss points of ${\overrightarrow{A_{1} A_{3}}}_{3} \overrightarrow{A_{1} B_{3}}, \overrightarrow{B_{1} A_{3}}, \overrightarrow{C_{1} B_{3}}$ (which implies $\int_{s} \mathbf{u}_{h} d \sigma=0$ ) may be written

$$
\begin{aligned}
\mathbf{u}_{1}+\mathbf{u}_{3} & =0 \\
9 \mathbf{u}_{1}+\mathbf{u}_{13} & =0 \\
9 \mathbf{u}_{3}+\mathbf{u}_{31} & =0 .
\end{aligned}
$$

Noticing that

$$
\int_{T} q d x=0 \Leftrightarrow 2\left(q_{1}+q_{2}+q_{3}\right)+q_{12}+q_{23}+q_{13}=0,
$$

the equation $\operatorname{div}_{h} \mathbf{u}_{h}=q$ on $T$ is equivalent to

$$
\begin{aligned}
3 \mathbf{u}_{1} \cdot \nabla \lambda_{1}+\mathbf{u}_{12} \cdot \nabla \lambda_{2}+\mathbf{u}_{13} \cdot \nabla \lambda_{3} & =q_{1}, \\
3 \mathbf{u}_{2} \cdot \nabla \lambda_{2}+\mathbf{u}_{23} \cdot \nabla \lambda_{3}+\mathbf{u}_{21} \cdot \nabla \lambda_{1} & =q_{2}, \\
3 \mathbf{u}_{3} \cdot \nabla \lambda_{3}+\mathbf{u}_{31} \cdot \nabla \lambda_{1}+\mathbf{u}_{32} \cdot \nabla \lambda_{2} & =q_{3}, \\
2 \mathbf{u}_{23} \cdot \nabla \lambda_{2}+2 \mathbf{u}_{32} \cdot \nabla \lambda_{3}+\mathbf{u}_{123} \cdot \nabla \lambda_{1} & =q_{23}, \\
2 \mathbf{u}_{31} \cdot \nabla \lambda_{3}+2 \mathbf{u}_{13} \cdot \nabla \lambda_{1}+\mathbf{u}_{123} \cdot \nabla \lambda_{2} & =q_{13} .
\end{aligned}
$$

Taking into account (3.2), continuity with 0 at the Gauss points of ${\overrightarrow{A_{2} B_{1}}}_{1}$ and ${\overrightarrow{A_{2}}}_{1}$ is equivalent to

$$
\begin{aligned}
\left(\mathbf{u}_{1}+\mathbf{u}_{2}\right)^{(k)}=0, & k=0,3 \\
\left(9 \mathbf{u}_{1}+\mathbf{u}_{12}\right)^{(k)} & =0, \quad k=0,3
\end{aligned}
$$


Using all of these equations, we now study $\operatorname{Ker}\left(\operatorname{div}_{h}\right)$. To do so, we set $q=0$. In triangle $T_{0}$ we obtain from (3.10), (3.11), (3.12), (3.2), (3.18), and (3.19) that

$$
\mathbf{u}_{2}=\mathbf{u}_{3}=-\mathbf{u}_{1}, \quad \mathbf{u}_{31}=\mathbf{u}_{21}=-\mathbf{u}_{13}=-\mathbf{u}_{12}=9 \mathbf{u}_{1} .
$$

We deduce from (3.13) that

$$
12 \mathbf{u}_{1} \cdot \nabla \lambda_{1}=0
$$

from (3.14) that

$$
\mathbf{u}_{23} \cdot \nabla \lambda_{3}=3 \mathbf{u}_{1} \cdot \nabla \lambda_{2}
$$

and from (3.15) that

$$
\mathbf{u}_{32} \cdot \nabla \lambda_{2}=3 \mathbf{u}_{1} \cdot \nabla \lambda_{3}
$$

Therefore,

$$
\left(9 \mathbf{u}_{2}+\mathbf{u}_{23}\right) \cdot \nabla \lambda_{3}=-9 \mathbf{u}_{1} \cdot \nabla \lambda_{3}+3 \mathbf{u}_{1} \cdot \nabla \lambda_{2}=12 \mathbf{u}_{1} \cdot \nabla \lambda_{2},
$$

and using (3.1),

$$
\left(9 \mathbf{u}_{2}+\mathbf{u}_{23}\right) \cdot \nabla \lambda_{2}=\left(6 \mathbf{u}_{2}-3 \mathbf{u}_{3}-\mathbf{u}_{32}\right) \cdot \nabla \lambda_{2}=0 .
$$

Let

$$
\alpha=\left(\mathbf{u}_{1} \cdot \nabla \lambda_{2}\right)^{(0)}
$$

Then

$$
\begin{aligned}
\left(\mathbf{u}_{2}+\mathbf{u}_{3}\right)^{(0)} & =2 \alpha \overrightarrow{A_{2} A}, \\
\left(9 \mathbf{u}_{2}+\mathbf{u}_{23}\right)^{(0)} & =12 \alpha \overrightarrow{B_{1} A_{3}} .
\end{aligned}
$$

In triangle $T_{1}$ we use (3.6) and (3.7) with $k=0$ to obtain

$$
\begin{aligned}
\left(\mathbf{u}_{2}+\mathbf{u}_{3}\right)^{(1)} & =2 \alpha \overrightarrow{A_{2} A_{3}}, \\
\left(9 \mathbf{u}_{2}+\mathbf{u}_{23}\right)^{(1)} & =12 \alpha \overrightarrow{B_{1} A_{3}},
\end{aligned}
$$

and using (3.1),

$$
\left(9 u_{3}+u_{32}\right)^{(1)}=-12 \alpha \overrightarrow{B_{1} A_{2}} .
$$

Next, we use (3.15) and (3.12) to get

$$
12\left(u_{3} \cdot \nabla \lambda_{3}-\alpha \vec{B}_{1} A_{2} \cdot \nabla \lambda_{2}\right)=0
$$

i.e.,

$$
\mathbf{u}_{3} \cdot \nabla \lambda_{3}=\alpha\left(1-\nu_{2}\right)
$$

Let

$$
\beta=\left(\mathbf{u}_{3} \cdot \nabla \lambda_{1}\right)^{(1)} .
$$

Then

$$
\mathbf{u}_{3}=\beta{\overrightarrow{A_{2} A}}_{1}+\alpha\left(1-\nu_{2}\right){\overrightarrow{A_{2} A_{3}}}
$$

and from (3.10),

$$
\mathbf{u}_{1}+\mathbf{u}_{2}=\mathbf{u}_{2}-\mathbf{u}_{3}=2 \alpha \overrightarrow{A_{2} A_{3}}-2 \mathbf{u}_{3}=-2\left(\beta \overrightarrow{A_{2} A_{1}}-\alpha \nu_{2} \overrightarrow{A_{2} A_{3}}\right)
$$


i.e.,

$$
\left(\mathbf{u}_{1}+\mathbf{u}_{2}\right)^{(1)}=-2\left(\beta{\overrightarrow{A_{2} A_{1}}}_{\left.-\alpha \nu_{2}{\overrightarrow{A_{2}}}_{3}\right)}\right.
$$

From (3.13) and (3.11) we obtain

$$
\begin{aligned}
\left(9 \mathbf{u}_{1}+\mathbf{u}_{12}\right) \cdot \nabla \lambda_{2} & =9 \mathbf{u}_{1} \cdot \nabla \lambda_{2}-3 \mathbf{u}_{1} \cdot \nabla \lambda_{1}-\mathbf{u}_{13} \cdot \nabla \lambda_{3} \\
& =-12 \mathbf{u}_{1} \cdot \nabla \lambda_{1}=12 \mathbf{u}_{3} \cdot \nabla \lambda_{1}=12 \beta .
\end{aligned}
$$

From (3.2), (3.10), (3.14), and (3.22),

$$
\begin{aligned}
\left(9 \mathbf{u}_{1}+\mathbf{u}_{12}\right) \cdot \nabla \lambda_{1}= & \left(6 \mathbf{u}_{1}-3 \mathbf{u}_{2}-\mathbf{u}_{21}\right) \cdot \nabla \lambda_{1} \\
= & \left(6 \mathbf{u}_{1}-3 \mathbf{u}_{2}\right) \cdot \nabla \lambda_{1}+3 \mathbf{u}_{2} \cdot \nabla \lambda_{2}+\mathbf{u}_{23} \cdot \nabla \lambda_{3} \\
= & \left(6 \mathbf{u}_{1}-3 \mathbf{u}_{2}\right) \cdot \nabla \lambda_{1}+3 \mathbf{u}_{2} \cdot \nabla \lambda_{2}-9 \mathbf{u}_{2} \cdot \nabla \lambda_{3}+12 \alpha \vec{B}_{1} A_{3} \cdot \nabla \lambda_{3} \\
= & 6 \mathbf{u}_{1} \cdot \nabla \lambda_{1}+6 \mathbf{u}_{2} \cdot\left(\nabla \lambda_{2}-\nabla \lambda_{3}\right)+12 \alpha\left(1-\nu_{3}\right) \\
= & -6 \mathbf{u}_{3} \cdot \nabla \lambda_{1}+12 \alpha \overrightarrow{A_{2} A_{3}} \cdot\left(\nabla \lambda_{2}-\nabla \lambda_{3}\right)-6 \mathbf{u}_{3} \cdot\left(\nabla \lambda_{2}-\nabla \lambda_{3}\right) \\
& +12 \alpha\left(1-\nu_{3}\right) \\
= & 12 \mathbf{u}_{3} \cdot \nabla \lambda_{3}-12 \alpha\left(1+\nu_{3}\right)=12 \alpha\left(\nu_{1}-1\right) .
\end{aligned}
$$

With (3.27) that gives

$$
\left(9 \mathrm{u}_{1}+\mathrm{u}_{12}\right)^{(1)}=12\left[\alpha\left(\nu_{1}-1\right){\overrightarrow{A_{3} A_{1}}}_{+\beta A_{3} A_{2}}\right] .
$$

Similarly, we obtain

$$
\begin{aligned}
\left(\mathbf{u}_{1}+\mathbf{u}_{2}\right)^{(2)} & =-2\left(\delta \overrightarrow{A_{2} A_{1}}-\gamma \tau_{2}{\overrightarrow{A_{2} B}}_{3}\right) \\
\left(9 \mathbf{u}_{1}+\mathbf{u}_{12}\right)^{(2)} & =12\left[\gamma\left(\tau_{1}-1\right) \overrightarrow{B_{3} A_{1}}+\delta \vec{B}_{3}\right]
\end{aligned}
$$

where

$$
\gamma=\left(\mathbf{u}_{1} \cdot \nabla \lambda_{2}\right)^{(3)}
$$

and

$$
\delta=\left(\mathbf{u}_{3} \cdot \nabla \lambda_{1}\right)^{(2)}
$$

We remark that

$$
\begin{aligned}
& {\overrightarrow{A_{2} B_{3}}}^{=}=\mu_{1}{\overrightarrow{A_{2} A_{1}}}+\mu_{3}{\overrightarrow{A_{2} A_{3}}} \\
& \overrightarrow{B_{3} A_{1}}=\left(1-\mu_{1}\right){\overrightarrow{A_{3} A_{1}}}^{-\mu_{2} \vec{A}_{3} A_{2}} \\
& \overrightarrow{B_{3} A_{2}}=-\mu_{1}{\overrightarrow{A_{3} A_{1}}}+\left(1-\mu_{2}\right){\overrightarrow{A_{3} A_{2}}}
\end{aligned}
$$

Thus Eq. (3.8) becomes (from (3.26) and (3.29))

$$
\begin{aligned}
& \beta=\delta-\tau_{2} \mu_{1} \gamma \\
& \nu_{2} \alpha=\tau_{2} \mu_{3} \gamma
\end{aligned}
$$

and Eq. (3.9) becomes

$$
\begin{aligned}
& \left(\nu_{1}-1\right) \alpha=\left(1-\mu_{1}\right)\left(\tau_{1}-1\right) \gamma-\mu_{1} \delta \\
& \beta=-\left(\tau_{1}-1\right) \mu_{2} \gamma+\left(1-\mu_{2}\right) \delta .
\end{aligned}
$$


We thus obtain the linear system

$$
\left(\begin{array}{cccc}
0 & 1 & \mu_{1} \tau_{2} & -1 \\
\nu_{2} & 0 & -\mu_{3} \tau_{2} & 0 \\
\nu_{1}-1 & 0 & -\left(\tau_{1}-1\right)\left(1-\mu_{1}\right) & \mu_{1} \\
0 & 1 & \mu_{2}\left(\tau_{1}-1\right) & -\left(1-\mu_{2}\right)
\end{array}\right)\left(\begin{array}{l}
\alpha \\
\beta \\
\gamma \\
\delta
\end{array}\right)=0
$$

The determinant of this linear system is

$$
D=-\left(\nu_{1}-1\right) \tau_{2} \mu_{3} \mu_{2}+\nu_{2}\left[\left(\tau_{1}-1\right) \mu_{2}-\mu_{1}^{2} \tau_{2}\right] .
$$

Therefore, if $D \neq 0$, we have $\alpha=\beta=\gamma=\delta=0$. We then deduce from (3.20) and (3.21) that $\mathbf{u}_{1}^{(0)}=0$. Consequently,

$$
u_{2}^{(0)}=u_{3}^{(0)}=u_{12}^{(0)}=u_{21}^{(0)}=u_{31}^{(0)}=u_{13}^{(0)}=u_{23}^{(0)}=0 .
$$

It then follows from (3.1) that $\mathbf{u}_{32}^{(0)}=0$ and from (3.16) and (3.17) that $\mathbf{u}_{123}^{(0)}=0$. Hence $\mathbf{u}_{h}^{(0)}=0$. In a similar way, we obtain $\mathbf{u}_{h}^{(1)}=\mathbf{u}_{h}^{(2)}=\mathbf{u}_{h}^{(3)}=0$, so that $\mathbf{u}_{h}=0$. Since this shows that $\operatorname{Ker}\left(\operatorname{div}_{h}\right)=\{0\}$, we get that $\operatorname{div}_{h}$ is one-to-one.

Finally, we consider the inequality stated in Proposition 3.1. To prove this result, we consider the previous equations with $q \neq 0$. First observe that Eqs. (3.1)-(3.15) give the values of

$$
\left(\mathbf{u}_{i} \cdot \nabla \lambda_{l}\right)^{(k)},\left(\mathbf{u}_{i j} \cdot \nabla \lambda_{l}\right)^{(k)}, \quad k=0,1,2,3
$$

as linear functions of $\left(q_{1}, q_{2}, q_{3}\right)^{\left(k^{\prime}\right)}, k^{\prime}=0,1,2,3$, and thus we obtain

$$
\begin{gathered}
\left|\left(\mathbf{u}_{i} \cdot \nabla \lambda_{l}\right)^{(k)}\right| \leq \frac{C_{1}}{|D|} \max _{i, k^{\prime}}\left|q_{i}^{\left(k^{\prime}\right)}\right|, \\
\left|\left(\mathbf{u}_{i j} \cdot \nabla \lambda_{l}\right)^{(k)}\right| \leq \frac{C_{2}}{|D|} \max _{i, k^{\prime}}\left|q_{i}^{\left(k^{\prime}\right)}\right| .
\end{gathered}
$$

We next use (3.16) and (3.17) to obtain

$$
\begin{aligned}
& \left|\left(\mathbf{u}_{123} \cdot \nabla \lambda_{1}\right)^{(k)}\right| \leq\left|q_{23}^{(k)}\right|+\frac{4 C_{2}}{|D|} \max _{i, k^{\prime}}\left|q_{i}^{\left(k^{\prime}\right)}\right| \\
& \left|\left(\mathbf{u}_{123} \cdot \nabla \lambda_{2}\right)^{(k)}\right| \leq\left|q_{13}^{(k)}\right|+\frac{4 C_{2}}{|D|} \max _{i, k^{\prime}}\left|q_{i}^{\left(k^{\prime}\right)}\right| .
\end{aligned}
$$

By standard scaling arguments, we have that

$$
\begin{aligned}
\left|q_{i}^{(k)}\right| & \leq C\|q\|_{L^{2}\left(T_{k}\right)} / A_{k}^{1 / 2} \\
\left|q_{i j}^{(k)}\right| & \leq C\|q\|_{L^{2}\left(T_{k}\right)} / A_{k}^{1 / 2} \\
\|\operatorname{Grad} \mathbf{u}\|_{L^{2}\left(T_{k}\right)} & \leq C(\theta)\left(\sum\left|\mathbf{u}_{i}\right|+\sum\left|\mathbf{u}_{i j}\right|+\left|\mathbf{u}_{123}\right|\right)
\end{aligned}
$$

where $A_{k}$ denotes the area of $T_{k}$. The inequality stated in Proposition 3.1 follows immediately.

In order to understand the condition on the determinant $D$ under which Proposition 3.1 is valid, we now examine this condition in more detail. For the configuration of triangles depicted in Figure 3.1, we have that $\nu_{1}<0, \mu_{3}<0, \tau_{1}<0$. If we assume that

$$
\nu_{2} \geq 0, \tau_{2} \geq 0, \mu_{2} \geq 0, \nu_{2}+\mu_{2} \neq 0, \nu_{2}+\tau_{2} \neq 0, \tau_{2}+\mu_{2} \neq 0,
$$


then it is easy to see that $D \neq 0$. Some meshes not covered by Proposition 1 are:

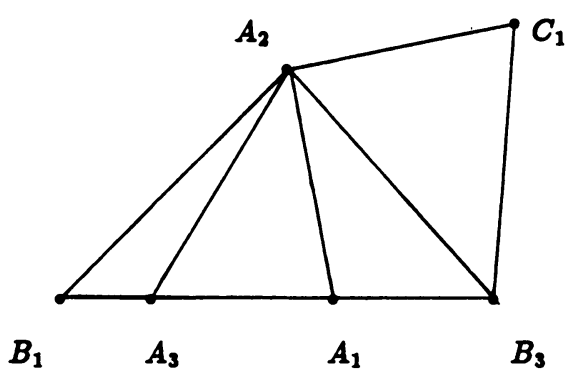

FIGURE 3.2. $\nu_{2}=\mu_{2}=0$

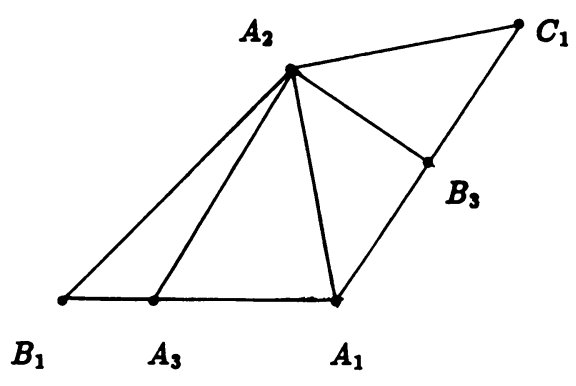

FIGURE 3.3. $\nu_{2}=\tau_{2}=0$

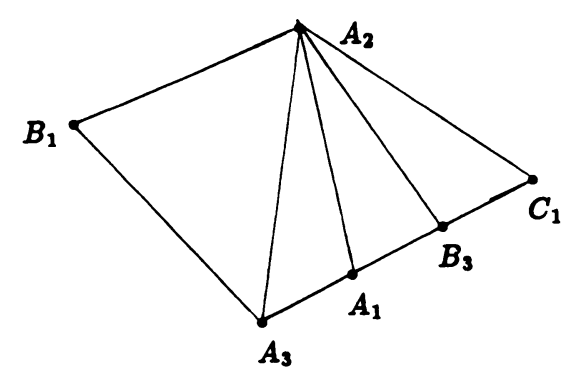

FIGURE 3.4. $\tau_{2}=\mu_{2}=0$

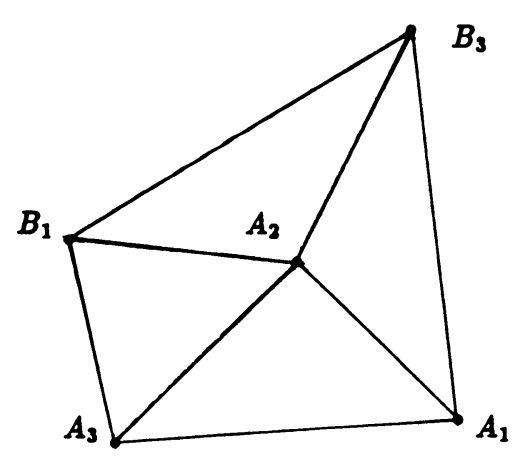

FIGURE 3.5 


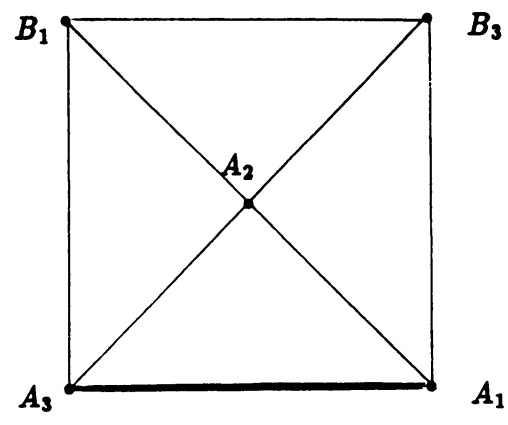

Figure 3.6

Remark. The limiting case $C_{1}=B_{1}$ is allowed. In this case, $\mathbf{u}_{h}=0$ at the Gauss points on $\overrightarrow{B_{1} A_{2}}$. Therefore, the mesh depicted in Figure 3.5 and, in particular, the special case depicted in Figure 3.6 are covered by Proposition 3.1.

4. Verification of Hypothesis H1-Mesh II. We next consider the mesh in Figure 4.1.

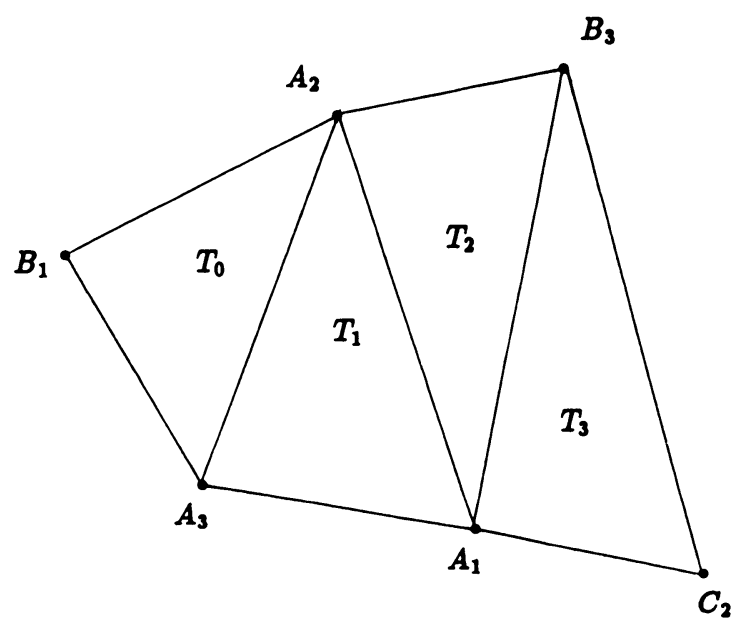

FIGURE 4.1

We use the same notation with the addition that $\left(\xi_{1}, \xi_{2}, \xi_{3}\right)$ are the barycentric coordinates of $C_{2}$ with respect to $A_{1}, A_{2}, B_{3}$. The verification of condition (2.5) is contained in the following proposition.

PROPOSITION 4.1. If

$$
\Delta=\left(\nu_{1}-1\right) \xi_{1} \mu_{2} \mu_{3}-\nu_{2}\left(\xi_{2}-1\right) \mu_{1}+\nu_{2} \xi_{1}\left[\left(1-\mu_{1}\right)^{2}-\mu_{3}\right] \neq 0
$$

then the operator $\operatorname{div}_{h}$ from $\hat{\mathbf{V}}_{h}$ into $H_{h}$ is one-to-one and for all $\mathbf{u}_{h} \in \hat{\mathbf{V}}_{h}$,

$$
\left\|\mathbf{u}_{h}\right\|_{1, h, \Omega_{r}} \leq \frac{C(\theta)}{|\Delta|}\left\|\operatorname{div}_{h} \mathbf{u}_{h}\right\|_{0, \Omega_{r}}
$$

Proof. We follow the proof of Proposition $3.1 \mathrm{up}$ to Eq. (3.27). In triangle $T_{1}$, it follows from (3.2) that

$$
9 \mathbf{u}_{2}+\mathbf{u}_{21}=6\left(\mathbf{u}_{2}+u_{1}\right)-9 u_{1}-u_{12}
$$


and from (3.26) and (3.27) that

$$
\left(9 \mathbf{u}_{2}+\mathbf{u}_{21}\right)^{(1)}=12\left\{\alpha \nu_{2}{\overrightarrow{A_{2}}}_{3}+\left[\alpha\left(\nu_{1}-1\right)+\beta\right] \overrightarrow{A_{1} A_{3}}\right\}
$$

By symmetry, we obtain

$$
\begin{aligned}
\left(\mathbf{u}_{1}+\mathbf{u}_{2}\right)^{(2)} & =-2\left[\delta{\overrightarrow{A_{1} A_{2}}}_{-\gamma \xi_{1}} \overrightarrow{A_{1} B_{3}}\right] \\
\left(9 \mathbf{u}_{1}+\mathbf{u}_{12}\right)^{(2)} & =12\left\{\gamma \xi_{1} \overrightarrow{A_{1} B_{3}}+\left[\gamma\left(\xi_{2}-1\right)+\delta\right] \overrightarrow{A_{2} B_{3}}\right\}
\end{aligned}
$$

Then (3.8) and (3.9) become

$$
\begin{aligned}
& \beta=-\delta-\gamma \xi_{1}\left(\mu_{1}-1\right), \\
& \nu_{2} \alpha=\xi_{1} \mu_{3} \gamma \\
& \left(\nu_{1}-1\right) \alpha=\left(\mu_{1}-1\right) \xi_{1} \gamma+\mu_{1}\left[\left(\xi_{2}-1\right) \gamma+\delta\right], \\
& \beta=\mu_{2} \xi_{1} \gamma+\left(\mu_{2}-1\right)\left[\left(\xi_{2}-1\right) \gamma+\delta\right] .
\end{aligned}
$$

We thus obtain the linear system

$$
\left(\begin{array}{cccc}
0 & 1 & \left(\mu_{1}-1\right) \xi_{1} & 1 \\
\nu_{2} & 0 & -\mu_{3} \xi_{1} & 0 \\
\nu_{1}-1 & 0 & \xi_{1}-\mu_{1}\left(\xi_{1}+\xi_{2}-1\right) & -\mu_{1} \\
0 & 1 & -\xi_{1} \mu_{2}-\left(\mu_{2}-1\right)\left(\xi_{2}-1\right) & \left(1-\mu_{2}\right)
\end{array}\right)\left(\begin{array}{l}
\alpha \\
\beta \\
\gamma \\
\delta
\end{array}\right)=0 .
$$

The remainder of the proof of Proposition 4.1 follows in an analogous fashion to that of Proposition 3.1.

We now examine the determinant $\Delta$ in more detail in order to understand the condition under which Proposition 4.1 is valid. For the configuration of triangles depicted in Figure 4.1, we have that $\nu_{1}<0, \mu_{3}<0, \xi_{2}<0$. If we assume that

$$
\xi_{1} \geq 0, \mu_{2} \geq 0, \mu_{1} \geq 0, \nu_{2} \geq 0, \nu_{2}+\xi_{1} \neq 0, \nu_{2}+\mu_{2} \neq 0, \xi_{1}+\mu_{1} \neq 0
$$

then it is easy to see that $\Delta \neq 0$. Some meshes not covered by Proposition 4.1 are

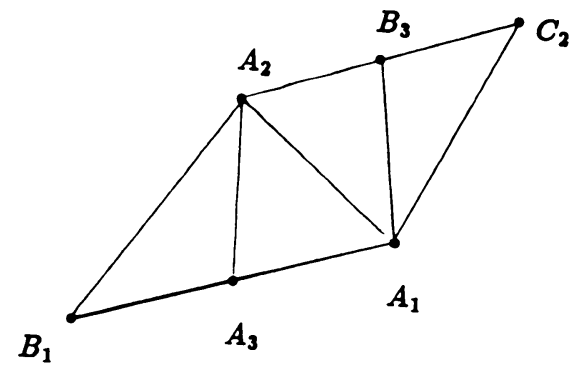

FIGURE 4.2. $\nu_{2}=\xi_{1}=0$ 


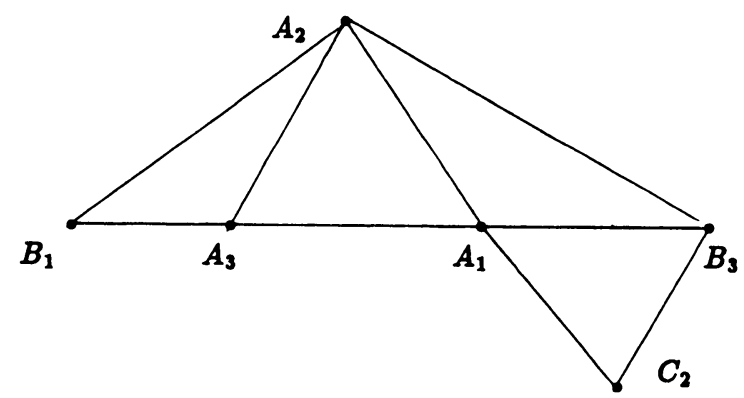

FIGURE 4.3. $\nu_{2}=\mu_{2}=0$

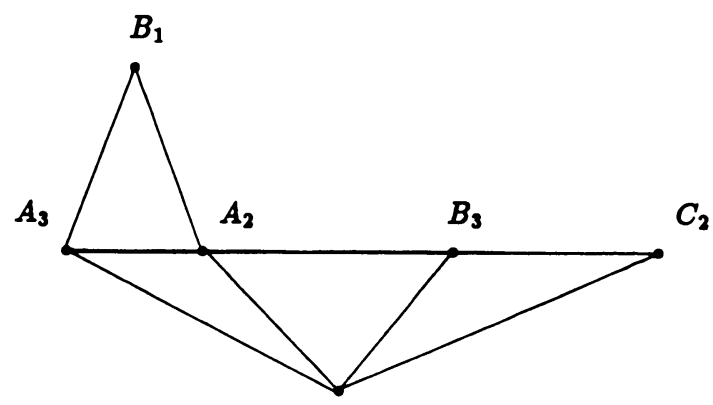

FIGURE 4.4. $\xi_{1}=\mu_{1}=0$

However, the standard regular mesh, depicted in Figure 4.5, is covered by Proposition 4.1.

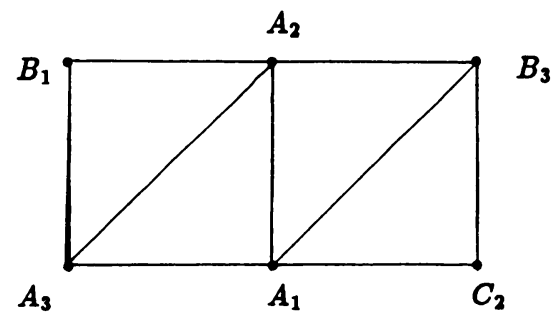

FIGURE 4.5

5. Verification of Hypothesis H1-Mesh III. Next, we turn to the case of three triangles aligned as in Figure 5.1.

We set $\Omega_{r}=T=T_{1} \cup T_{2} \cup T_{3}$. Let $\left(\nu_{1}, \nu_{2}, \nu_{3}\right)$ denote the barycentric coordinates of $B$ with respect to the points $A_{1}, A_{2}, A_{3}$ and let $\left(\mu_{1}, \mu_{2}, \mu_{3}\right)$ denote the barycentric coordinates of a point $x \in T$ with respect to $A_{1}, A_{2}, A_{3}$. To define the barycentric coordinates in each $T_{i}$, we also denote the vertex $B$ in the triangle $T_{i}$ by $B_{i}$. We then denote by $\lambda_{j}^{(i)}$ the barycentric coordinate of a point $x \in T_{i}$ with respect to the vertex of $T_{i}$ with subscript $j$. Define the subspace $\mathbf{X}_{h}$ of $\mathbf{V}_{h}$ by

$$
\mathbf{X}_{h}=\left\{\mathbf{u}_{h} \in\left(C^{0}\left(\mathbf{R}^{2}\right)\right)^{2}:\left.\mathbf{u}_{h}\right|_{T_{i}} \in\left(P_{3}\right)^{2}, \mathbf{u}_{h}(B)=0, \mathbf{u}_{h}=0 \text { in } \Omega / T\right\} .
$$




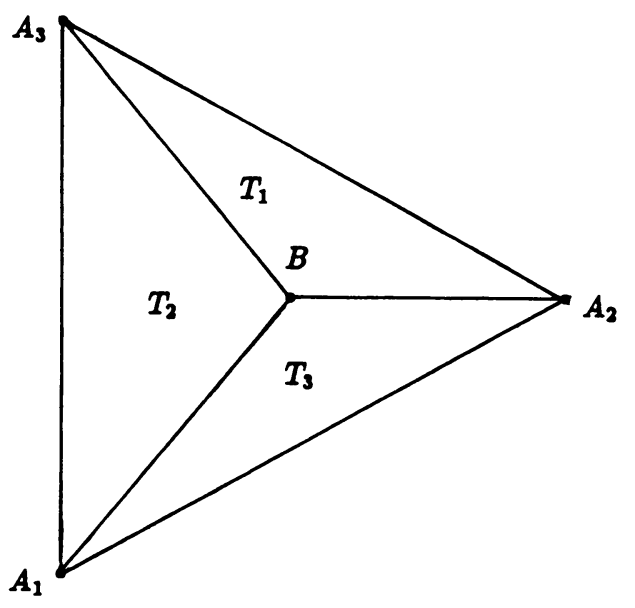

FIGURE 5.1

Note that $\mathbf{u}_{h} \in \mathbf{X}_{h}$ may be represented in the triangle $T_{i}$ by

$$
\mathbf{u}_{h}=\sum_{\substack{j=1 \\ j \neq i}}^{3} \mathbf{u}_{i j}^{(i)} \lambda_{i}^{2} \lambda_{j}+\sum_{\substack{j=1 \\ j \neq i}}^{3} \mathbf{u}_{j i}^{(i)} \lambda_{j}^{2} \lambda_{i}+\mathbf{u}_{123}^{(i)} \lambda_{1} \lambda_{2} \lambda_{3},
$$

where we have omitted the superscript $(i)$ on the barycentric coordinates. We will continue to omit superscripts (on $\mathbf{u}_{i j}$ as well) when there is no ambiguity about the triangle. Using the continuity of $\mathbf{u}_{h}$, it follows by comparing the expressions for $\mathbf{u}_{h}$ along common interior sides, that

$$
\begin{array}{ll}
\mathbf{u}_{13}^{(1)}=\mathbf{u}_{23}^{(2)}, & \mathbf{u}_{31}^{(1)}=\mathbf{u}_{32}^{(2)}, \\
\mathbf{u}_{12}^{(2)}=\mathbf{u}_{13}^{(3)}, & \mathbf{u}_{21}^{(2)}=\mathbf{u}_{31}^{(3)}, \\
\mathbf{u}_{23}^{(3)}=\mathbf{u}_{21}^{(1)}, & \mathbf{u}_{32}^{(3)}=\mathbf{u}_{12}^{(1)} .
\end{array}
$$

Using the above, it is easy to check that $\operatorname{dim} \mathbf{X}_{h}=18$, while $H_{h}$, defined in Section 2, has dimension 15. To get a result analogous to Propositions 3.1 and 4.1, we define

$$
\hat{\mathbf{V}}_{h}=\left\{\mathbf{u}_{h} \in \mathbf{X}_{h}: \int_{s} \mathbf{u}_{h} \cdot \nu d \sigma=0, \text { for all sides } s \text { of } \Omega_{r}\right\}
$$

where $\nu$ is the normal to $s$. Note that $\int_{s} \mathbf{u}_{h} \cdot \nu d \sigma=0$ is equivalent to the conditions

$$
\begin{aligned}
& \left(\mathbf{u}_{31}^{(1)}+\mathbf{u}_{13}^{(1)}\right) \cdot \nabla \lambda_{2}^{(1)}=\left(\mathbf{u}_{21}^{(1)}+\mathbf{u}_{12}^{(1)}\right) \cdot \nabla \lambda_{3}^{(1)}=0 \\
& \left(\mathbf{u}_{12}^{(2)}+\mathbf{u}_{21}^{(2)}\right) \cdot \nabla \lambda_{3}^{(2)}=\left(\mathbf{u}_{32}^{(2)}+\mathbf{u}_{23}^{(2)}\right) \cdot \nabla \lambda_{1}^{(2)}=0 \\
& \left(\mathbf{u}_{32}^{(3)}+\mathbf{u}_{23}^{(3)}\right) \cdot \nabla \lambda_{1}^{(3)}=\left(\mathbf{u}_{13}^{(3)}+\mathbf{u}_{31}^{(3)}\right) \cdot \nabla \lambda_{2}^{(3)}=0 .
\end{aligned}
$$

We will then prove:

PROPOSITION 5.1. The operator div from $\hat{\mathbf{V}}_{h}$ into $H_{h}$ is one-to-one and for all $\mathbf{u}_{h} \in \hat{\mathbf{V}}_{h}$,

$$
\left\|\mathbf{u}_{h}\right\|_{1, h, \Omega_{r}} \leq C(\theta)\left\|\operatorname{div}_{h} \mathbf{u}_{h}\right\|_{0, \Omega_{r}}
$$


Note that for this mesh, we are actually proving the inf-sup condition for conforming piecewise cubics.

Before proving Proposition 5.1, we gather some facts about the relationships among the various barycentric coordinates defined above. By writing $B$ as a convex combination of the $A_{i}$, we easily obtain

$$
\begin{aligned}
& \mu_{1}=\nu_{1} \lambda_{1}^{(1)}=\nu_{1} \lambda_{2}^{(2)}+\lambda_{1}^{(2)}=\nu_{1} \lambda_{3}^{(3)}+\lambda_{1}^{(3)}, \\
& \mu_{2}=\nu_{2} \lambda_{1}^{(1)}+\lambda_{2}^{(1)}=\nu_{2} \lambda_{2}^{(2)}=\nu_{2} \lambda_{3}^{(3)}+\lambda_{2}^{(3)}, \\
& \mu_{3}=\nu_{3} \lambda_{1}^{(1)}+\lambda_{3}^{(1)}=\nu_{3} \lambda_{2}^{(2)}+\lambda_{3}^{(2)}=\nu_{3} \lambda_{3}^{(3)} .
\end{aligned}
$$

From these relations it follows that

$$
\nu_{3} \nabla \lambda_{3}^{(1)}=-\nu_{1} \nabla \lambda_{1}^{(3)}, \quad \nu_{1} \nabla \lambda_{2}^{(1)}=-\nu_{2} \nabla \lambda_{1}^{(2)}, \quad \nu_{2} \nabla \lambda_{3}^{(2)}=-\nu_{3} \nabla \lambda_{2}^{(3)}
$$

By first expressing $\lambda_{i}^{(j)}$ as a linear function of $\mu_{1}, \mu_{2}, \mu_{3}$ and then using the fact that $\sum_{i=1}^{3} \nabla \lambda_{i}^{(j)}=0$, we obtain the further relations

$$
\begin{aligned}
& \nabla \lambda_{2}^{(1)}=\left(\nabla \mu_{2}+\nu_{2} \nabla \lambda_{3}^{(1)}\right) /\left(1-\nu_{2}\right), \\
& \nabla \lambda_{3}^{(1)}=\left(\nabla \mu_{3}+\nu_{3} \nabla \lambda_{2}^{(1)}\right) /\left(1-\nu_{3}\right), \\
& \nabla \lambda_{3}^{(2)}=\left(\nabla \mu_{3}+\nu_{3} \nabla \lambda_{1}^{(2)}\right) /\left(1-\nu_{3}\right), \\
& \nabla \lambda_{1}^{(2)}=\left(\nabla \mu_{1}+\nu_{1} \nabla \lambda_{3}^{(2)}\right) /\left(1-\nu_{1}\right), \\
& \nabla \lambda_{1}^{(3)}=\left(\nabla \mu_{1}+\nu_{1} \nabla \lambda_{2}^{(3)}\right) /\left(1-\nu_{1}\right), \\
& \nabla \lambda_{2}^{(3)}=\left(\nabla \mu_{2}+\nu_{2} \nabla \lambda_{1}^{(3)}\right) /\left(1-\nu_{2}\right) .
\end{aligned}
$$

Using these facts, we are now ready to prove Proposition 5.1.

Proof of Proposition 5.1. From the equation div $\mathbf{u}_{h}=q$, we get in triangle $T_{1}$ the equations

$$
\begin{aligned}
\mathbf{u}_{12} \cdot \nabla \lambda_{2}+\mathbf{u}_{13} \cdot \nabla \lambda_{3} & =q_{1}, \\
\mathbf{u}_{21} \cdot \nabla \lambda_{1} & =q_{2}, \\
\mathbf{u}_{31} \cdot \nabla \lambda_{1} & =q_{3}, \\
2 \mathbf{u}_{12} \cdot \nabla \lambda_{1}+2 \mathbf{u}_{21} \cdot \nabla \lambda_{2}+\mathbf{u}_{123} \cdot \nabla \lambda_{3} & =q_{12}, \\
\mathbf{u}_{123} \cdot \nabla \lambda_{1} & =q_{23},
\end{aligned}
$$

in triangle $T_{2}$ the equations

$$
\begin{aligned}
\mathbf{u}_{12} \cdot \nabla \lambda_{2} & =q_{1}, \\
\mathbf{u}_{21} \cdot \nabla \lambda_{1}+\mathbf{u}_{23} \cdot \nabla \lambda_{3} & =q_{2}, \\
\mathbf{u}_{32} \cdot \nabla \lambda_{2} & =q_{3}, \\
\mathbf{u}_{123} \cdot \nabla \lambda_{2} & =q_{13}, \\
2 \mathbf{u}_{23} \cdot \nabla \lambda_{2}+2 \mathbf{u}_{32} \cdot \nabla \lambda_{3}+\mathbf{u}_{123} \cdot \nabla \lambda_{1} & =q_{23},
\end{aligned}
$$


and in triangle $T_{3}$ the equations

$$
\begin{aligned}
\mathbf{u}_{13} \cdot \nabla \lambda_{3} & =q_{1}, \\
\mathbf{u}_{23} \cdot \nabla \lambda_{3} & =q_{2}, \\
\mathbf{u}_{31} \cdot \nabla \lambda_{1}+\mathbf{u}_{32} \cdot \nabla \lambda_{2} & =q_{3}, \\
\mathbf{u}_{123} \cdot \nabla \lambda_{3} & =q_{12}, \\
2 \mathbf{u}_{31} \cdot \nabla \lambda_{3}+2 \mathbf{u}_{13} \cdot \nabla \lambda_{1}+\mathbf{u}_{123} \cdot \nabla \lambda_{1} & =q_{13} .
\end{aligned}
$$

Now observe that $\mathbf{u}_{21}^{(1)}=\mathbf{u}_{23}^{(3)}$ is determined from Eqs. (5.13) and (5.23), $\mathbf{u}_{31}^{(1)}=$ $\mathbf{u}_{32}^{(2)}$ is determined form Eqs. (5.14) and (5.19), and $\mathbf{u}_{12}^{(2)}=\mathbf{u}_{13}^{(3)}$ is determined from Eqs. (5.17) and (5.22). More precisely, using the relationships among the barycentric coordinates, the functions $\mathbf{u}_{j i}^{(i)} \cdot \nabla \lambda_{k}^{(i)}, i=1,3, j=1,3, k=1,3, j \neq i$, may be written as linear combinations of $q_{j}^{(i)}, i=1,3, j=1,3$, and these $\mathbf{u}_{j i}^{(i)}=0$ if the above $q_{j}^{(i)}=0$. Then $\mathbf{u}_{i j}^{(i)} \cdot \nabla \lambda_{k}^{(i)}, i \neq j \neq k$, is determined by Eqs. (5.2), (5.3), and (5.4). We deduce from (5.6), (5.7), and (5.12) that in triangle $T_{1}$

$$
\begin{aligned}
& \frac{1}{1-\nu_{2}} \mathbf{u}_{12} \cdot \nabla \mu_{2}+\frac{1}{1-\nu_{3}} \mathbf{u}_{13} \cdot \nabla \mu_{3} \\
& \quad=q_{1}-\frac{\nu_{2}}{1-\nu_{2}} \mathbf{u}_{12} \cdot \nabla \lambda_{3}^{(1)}-\frac{\nu_{3}}{1-\nu_{3}} \mathbf{u}_{13} \cdot \nabla \lambda_{2}^{(1)} .
\end{aligned}
$$

Similarly, in $T_{2}$

$$
\begin{aligned}
& \frac{1}{1-\nu_{3}} \mathbf{u}_{23} \cdot \nabla \mu_{3}+\frac{1}{1-\nu_{1}} \mathbf{u}_{21} \cdot \nabla \mu_{1} \\
& \quad=q_{2}-\frac{\nu_{3}}{1-\nu_{3}} \mathbf{u}_{23} \cdot \nabla \lambda_{1}^{(2)}-\frac{\nu_{1}}{1-\nu_{1}} \mathbf{u}_{21} \cdot \nabla \lambda_{3}^{(2)}
\end{aligned}
$$

and in $T_{3}$

$$
\begin{aligned}
& \frac{1}{1-\nu_{1}} \mathbf{u}_{31} \cdot \nabla \mu_{1}+\frac{1}{1-\nu_{2}} \mathbf{u}_{32} \cdot \nabla \mu_{2} \\
& \quad=q_{3}-\frac{\nu_{1}}{1-\nu_{1}} \mathbf{u}_{31} \cdot \nabla \lambda_{2}^{(3)}-\frac{\nu_{2}}{1-\nu_{2}} \mathbf{u}_{32} \cdot \nabla \lambda_{1}^{(3)} .
\end{aligned}
$$

Using the equivalences in (5.1), the above system of equations may be reduced to a linear system in the three unknowns $\mathbf{u}_{12}^{(1)} \cdot \nabla \mu_{2}, \mathbf{u}_{23}^{(2)} \cdot \nabla \mu_{3}, \mathbf{u}_{31}^{(3)} \cdot \nabla \mu_{1}$, with matrix

$$
\left(\begin{array}{ccc}
\left(1-\nu_{2}\right)^{-1} & \left(1-\nu_{3}\right)^{-1} & 0 \\
0 & \left(1-\nu_{3}\right)^{-1} & \left(1-\nu_{1}\right)^{-1} \\
\left(1-\nu_{2}\right)^{-1} & 0 & \left(1-\nu_{1}\right)^{-1}
\end{array}\right)
$$

The determinant of this matrix is $2\left[\left(1-\nu_{1}\right)\left(1-\nu_{2}\right)\left(1-\nu_{3}\right)\right]^{-1} \neq 0$, which allows us to determine $\mathbf{u}_{i j}^{(i)}$. Finally, we use Eqs. (5.15), (5.16), (5.20), (5.21), (5.25), and (5.26) to determine $\mathbf{u}_{123}^{(i)}$ as linear functions of the $q_{i j}$ and $q_{i}$. Thus we see that the operator div from $\hat{\mathbf{V}}_{h}$ into $H_{h}$ is one-to-one. Inequality (5.5) follows by the same scaling argument used in the proof of Proposition 3.1.

6. Additional Results. In checking the validity of Hypothesis $\mathrm{H} 1$ for a given mesh, it may be the case that there are a few triangles which do not easily fit into one of the mesh types covered in the previous sections. In that case, the following lemmas may be useful. 
LEMMA 6.1. Let $T_{0}$ and $T_{1}$ be two triangles with a common side $s$ and $\Omega_{0}=$ $T_{0} \cup T_{1}$. Then, for all $q_{h} \in H_{h}\left(T_{0}\right)$, there exists $\mathbf{u}_{h} \in \mathrm{V}_{h}\left(\Omega_{0}\right)$ such that

$$
\operatorname{div}_{h} \mathbf{u}_{h}=q_{h} \quad \text { in } T_{0}, \quad \int_{s} \mathbf{u}_{h} d \sigma=0, \quad \text { and } \quad\left\|\mathbf{u}_{h}\right\|_{1, h, \Omega_{0}} \leq C(\theta)\left\|q_{h}\right\|_{0, T_{0}}
$$

where $C$ depends only on the smallest angle $\theta$ of the triangles $T_{0}$ and $T_{1}$.

Proof.

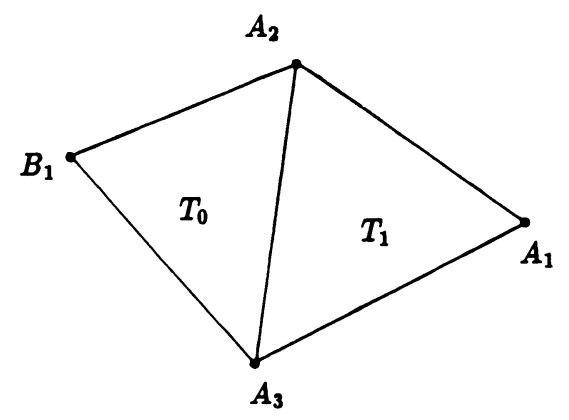

FiguRE 6.1

Using the notation of Proposition 3.1 and Figure 6.1, we see that the requirement that $\mathbf{u}^{(0)}=0$ at the Gauss points of $\overrightarrow{B_{1} A_{2}}$ and $\overrightarrow{B_{1} A_{3}}$ leads to the conditions

$$
\mathbf{u}_{2}=\mathbf{u}_{3}=-\mathbf{u}_{1}, \quad \mathbf{u}_{13}=-\mathbf{u}_{31}=-9 \mathbf{u}_{1}, \quad \mathbf{u}_{12}=-\mathbf{u}_{21}=-9 \mathbf{u}_{1},
$$

and the requirement that $\int_{s} \mathbf{u}_{h} d \sigma=0$ on ${\overrightarrow{A_{3} A_{2}}}_{2}$ can be implemented by setting

$$
\mathbf{u}_{23}=3 \mathbf{u}_{1}+\kappa, \quad \mathbf{u}_{32}=3 \mathbf{u}_{1}-\kappa
$$

with $\kappa$ to be determined. Setting $\alpha=\mathbf{u}_{1} \cdot \nabla \lambda_{2}$, the equation $\operatorname{div}_{h} \mathbf{u}_{h}=q_{h}$ in $T_{0}$ leads to the equations

$$
\begin{aligned}
\mathbf{u}_{1} \cdot \nabla \lambda_{1} & =q_{1} / 12, \\
\kappa \cdot \nabla \lambda_{3} & =q_{2}-q_{1} / 2+6 \alpha, \\
\kappa \cdot \nabla \lambda_{2} & =-q_{3}+q_{1}+6 \alpha, \\
\mathbf{u}_{123} \cdot \nabla \lambda_{2} & =q_{13}+3 q_{1}+18 \alpha, \\
\mathbf{u}_{123} \cdot \nabla \lambda_{3} & =q_{12}+3 q_{1} / 2-18 \alpha .
\end{aligned}
$$

It is easy to check that choosing $\alpha=-q_{1} / 24$ and defining $u$ in triangle $T_{1}$ by

$$
\mathbf{u}_{i}^{(1)}=\mathbf{u}_{i}^{(0)}, \quad \mathbf{u}_{i j}^{(1)}=\mathbf{u}_{i j}^{(0)}, \quad \mathbf{u}_{123}^{(1)}=\mathbf{u}_{123}^{(0)}
$$

gives the desired result.

LEMMA 6.2. If $\bar{\Omega}_{s}=\bar{\Omega}_{r} \cup T$, where $T$ has a common side with $\Omega_{r}$, and if Hypothesis $\mathrm{H} 1$ holds for $\Omega_{r}$, then Hypothesis $\mathrm{H} 1$ holds for $\Omega_{s}$.

Proof. Let $q_{h} \in H_{h}\left(\Omega_{s}\right)$. Lemma 6.1 then implies that there exists $\mathbf{u}_{0} \in \mathbf{V}_{h}\left(\Omega_{s}\right)$ such that

$$
\operatorname{div}_{h} \mathbf{u}_{0}=q_{h} \text { in } T \text { and }\left\|\mathbf{u}_{0}\right\|_{1, h, \Omega} \leq C_{1}\left\|q_{h}\right\|_{0, \Omega_{s}} .
$$

Setting $q_{1}=q_{h}-\operatorname{div}_{h} \mathbf{u}_{0}$, we observe that $\int_{s} \mathbf{u}_{h} d \sigma=0$ implies that $q_{1} \in H_{h}\left(\Omega_{r}\right)$. 
Hence, there exists $\mathbf{u}_{1} \in \mathbf{V}_{h}\left(\Omega_{r}\right)$ with $\operatorname{div}_{h} \mathbf{u}_{1}=q_{1}$ and

$$
\left\|\mathbf{u}_{1}\right\|_{1, h, \Omega_{s}} \leq\left\|q_{1}\right\|_{0, \Omega_{r}} / \gamma_{r} \leq\left(1+C_{1} \sqrt{2}\right)\left\|q_{h}\right\|_{0, \Omega_{s}} / \gamma_{r} .
$$

Taking $\mathbf{u}_{h}=\mathbf{u}_{0}+\mathbf{u}_{1}$, we get that $\operatorname{div}_{h} \mathbf{u}_{h}=q_{h}$ and

$$
\left\|\mathbf{u}_{h}\right\|_{1, h, \Omega_{s}} \leq\left(C_{1}+\left[1+C_{1} \sqrt{2}\right] / \gamma_{r}\right)\left\|q_{h}\right\|_{0, \Omega_{s}}=\left\|q_{h}\right\|_{0, \Omega_{s}} / \gamma_{s},
$$

with $\gamma_{s}=\gamma_{r} /\left(1+C_{1} \sqrt{2}+C_{1} \gamma_{r}\right)$.

Remark. Finally, since we have not found a counterexample, we conjecture that the spaces $\mathbf{W}_{h}$ and $Q_{h}$ form a stable Stokes pair for any triangulation of a convex polygon satisfying the minimal angle condition and containing an interior vertex.

Mathématiques-IRISA

Université de Rennes I

Campus de Beaulieu

35042 Rennes Cedex, France

crouzeix@parasol.irisa.fr

Department of Mathematics

Rutgers University

New Brunswick, New Jersey 08903

falk@fermat.rutgers.edu

1. C. BERnARDi \& G. RAUGEL, "Analysis of some finite elements for the Stokes problem," Math. Comp., v. 44, 1985, pp. 71-79.

2. J. BOLAND \& R. NICOLAIDES, "Stability of finite elements under divergence constraints," SIAM J. Numer. Anal., v. 20, 1983, pp. 722-731.

3. F. BREZZI, "On the existence, uniqueness, and the approximation of saddle point problems arising from Lagrangian multipliers," RAIRO Anal. Numér., v. 8, 1974, pp. 129-151.

4. M. CROUZEIX \& P.-A. RAVIART, "Conforming and non-conforming finite element methods for solving the stationary Stokes equations," RAIRO Anal. Numér., v. 7, 1973, pp. 33-76.

5. M. Fortin, Calcul Numérique des Écoulements des Fluides de Bingham et des Fluides Newtoniens Incompressibles par la Méthode des Éléments Finis, Thèse Univ. Paris, 1972.

6. M. FORTIN, "Old and new finite elements for incompressible flows," Internat. J. Numer. Methods Fluids, v. 1, 1981, pp. 347-364.

7. M. FORTIN, "A non-conforming piecewise quadratic finite element on triangles," Internat. J. Numer. Methods Engrg., v. 19, 1983, pp. 505-520.

8. V. GIRAULT \& P.-A. RAVIART, Finite Element Methods for Navier-Stokes Equations, SpringerVerlag, Berlin, 1986.

9. P.-A. Raviart \& J. M. Thomas, "Primal hybrid finite element methods for 2nd order elliptic equations," Math. Comp., v. 31, 1977, pp. 391-413.

10. L. R. SCOTT \& M. VOGELIUS, "Norm estimates for a maximal right inverse of the divergence operator in spaces of piecewise polynomials," Math. Modelling and Numer. Anal., v. 19, 1985, pp. 111-143.

11. R. STENBERG, "Analysis of mixed finite element methods for the Stokes problem: A unified approach," Math. Comp., v. 42, 1984, pp. 9-23. 